\title{
Nonrigid Brain MR Image Registration Using Uniform Spherical Region Descriptor
}

\author{
Shu Liao and Albert C. S. Chung
}

\begin{abstract}
There are two main issues that make nonrigid image registration a challenging task. First, voxel intensity similarity may not be necessarily equivalent to anatomical similarity in the image correspondence searching process. Second, during the imaging process, some interferences such as unexpected rotations of input volumes and monotonic gray-level bias fields can adversely affect the registration quality. In this paper, a new feature-based nonrigid image registration method is proposed. The proposed method is based on a new type of image feature, namely, uniform spherical region descriptor (USRD), as signatures for each voxel. The USRD is rotation and monotonic gray-level transformation invariant and can be efficiently calculated. The registration process is therefore formulated as a feature matching problem. The USRD feature is integrated with the Markov random field labeling framework in which energy function is defined for registration. The energy function is then optimized by the $\alpha$-expansion algorithm. The proposed method has been compared with five state-of-the-art registration approaches on both the simulated and real 3-D databases obtained from the BrainWeb and Internet Brain Segmentation Repository, respectively. Experimental results demonstrate that the proposed method can achieve high registration accuracy and reliable robustness behavior.
\end{abstract}

Index Terms-Monotonic gray-level transformation invariant, nonrigid image registration, rotation invariant, uniform spherical region descriptor (USRD).

\section{INTRODUCTION}

$\mathbf{N}$ ONRIGID IMAGE registration plays an important role in medical image analysis and processing. Its applications include, but are not limited to, anatomical analysis, brain disease diagnosis, and image data mining. Nonrigid image registration methods can be broadly classified into three categories: landmark-, intensity-, and feature-based registration methods. Landmark-based registration methods [1]-[4] first select a set of reliable landmarks from the input images. The landmark selection can be manually, semiautomatically, or even fully automatically performed. Features are extracted from those landmark points. The optimal transformations are then estimated based on these extracted features. Intensity-based approaches [5]-[8] define similarity function solely based on the joint intensity distri-

Manuscript received September 17, 2010; revised March 01, 2011; accepted May 21, 2011. Date of publication June 16, 2011; date of current version December 16, 2011. This work was supported by the K. S. Lo Foundation, Hong Kong. The associate editor coordinating the review of this manuscript and approving it for publication was Prof. Birsen Yazici.

The authors are with the Lo Kwee-Seong Medical Image Analysis Laboratory, Department of Computer Science and Engineering, The Hong Kong University of Science and Technology, Kowloon, Hong Kong (e-mail: liaoshu.cse@gmail.com; achung@cse.ust.hk).

Color versions of one or more of the figures in this paper are available online at http://ieeexplore.ieee.org.

Digital Object Identifier 10.1109/TIP.2011.2159615 butions of the template and subject images to guide the registration. The registration problem is thus formulated as an optimization process to minimize or maximize the target similarity functions. Feature-based approaches use anatomical features as signatures for each voxel, and then, registration is formulated as a feature matching process. Each stream of methods has its advantages and disadvantages. Landmark-based methods are usually computationally efficient as these methods include prior knowledge extracted from the selected landmarks. However, to obtain accurate registration results, a lot of reliable landmarks are required, and this can bring additional computational burdens. Intensity-based approaches are usually fully automatic. However, intensity similarity may not be necessarily equivalent to anatomical similarity. Feature-based approaches drive the registration process based on the adopted features. For instance, the tensor scale-based image features are used by [9] and [10] to rigidly align images acquired by different modalities. Nyul $e t$ $a l$. [11] extract the local-scaled-based features from each voxel to drive the registration process. Bagci and Bai [12] calculate the image features in the edgeless domain, and input images are first registered in the edgeless domain. Then, further alignment is performed in the intensity domain to the images registered in the edgeless domain. The intensity standardization procedure proposed in [13] is adopted in [12] as a preprocessing step to remove the effect of intensity gray-level differences. The hierarchical attribute matching mechanism for elastic registration (HAMMER) algorithm proposed by Shen and Davatzikos in [14] uses the geometric moment invariant (GMI) feature as voxel signature, and the robust image registration algorithm proposed by Yang et al. [15] represents the anatomical properties of each voxel by using the SIFT features.

There are two main challenging issues in nonrigid image registration. The first issue is that intensity similarity may not be necessarily equivalent to anatomical similarity in the image registration process. As pointed out in [16], using intensity distributions alone cannot fully characterize the anatomical differences between different tissues. Therefore, it is necessary to design effective anatomical region descriptor for defining signatures for each voxel. The second issue is that interferences such as unexpected volume rotation and monotonic gray-level bias fields generally exist during the imaging acquisition process. As such, the registration algorithm should be also robust against such interferences. As stated in [14], rotation invariance is a desired property for feature-based registration methods. Additionally, as pointed out in [17], the registration algorithm may prefer to align the bias fields between the template and subject images, instead of aligning their anatomical structures if the registration algorithm is not robust against bias fields. Noise in the input images can also degrade the performance of registration algorithms, as 
pointed out in [18]. The effect of noise is usually overcome by using filtering techniques during the registration procedure to suppress the noise information contained in the input images.

Therefore, we are motivated to propose a new feature-based nonrigid image registration method. The proposed method is based on a new anatomical region descriptor, namely, uniform spherical region descriptor (USRD). This paper is an extension of our initial work from [19] and [20]. The USRD encodes the anatomical information around each voxel, and it is rotation and monotonic gray-level transformation invariant. The USRD is integrated with the Markov random field (MRF) labeling framework to drive the registration process in this paper. The energy function of the MRF framework is optimized via the $\alpha$-expansion algorithm. The proposed method is evaluated in two different ways in this paper. First, the USRD feature is directly compared with other image features by using the Fisher separation criteria (FSC) [21] for registration task. The features for comparison are as follows: 1) voxel intensity used in free-form deformation (FFD) [22] and Demons [23]; 2) the GMI feature used in HAMMER [14]; 3) local-histogram-based feature (LHF) used in an extension of HAMMER [24]; and 4) the symmetric Alpha-stable filtered-based features used in [25]. Second, the proposed method has been also compared with five state-of-the-art registration approaches on both the simulated and real 3-D databases obtained from BrainWeb and Internet Brain Segmentation Repository (IBSR) based on the final registration accuracy under different conditions. It is shown that the proposed method achieves the highest registration accuracy among all the compared methods.

\section{USRD}

Here, we introduce the new image region descriptor, namely, the USRD. The USRD is a 2-D joint histogram consisting of two kinds of features: 1) the uniform spherical structure pattern (USSP) and 2) the uniform gradient spherical pattern (UGSP). The USSP and the UGSP encode the first- and second-order voxel information, respectively. We will also describe the USSP and the UGSP in detail and analyze their properties. Finally, we will show how to use the USRD to extract rotation and monotonic gray-level transformation invariant features from the input images.

\section{A. USSP}

The USSP feature is motivated from the idea of local binary patterns (LBPs) proposed in [26], which is an image feature originally designed for 2-D texture classification [26]. The USSP is a 3-D region descriptor that encodes the first-order image voxel interaction information. It also preserves the rotation and monotonic gray-level transformation invariant properties of LBPs.

Given input image $G$, for each voxel $v \in G$, spherical neighborhood $S_{v}$ centered at $v$ with radius $R$ is defined. $N_{v}$ samples are taken on the surface of $S_{v}$. In this paper, the deterministic incremental grid sequence sampling method proposed in [27] is adopted as the sampling method, which is uniform and computationally efficient.
For each sample $v_{i}$ on the surface of $S_{v}$, it is converted to binary number $B_{i}$ by comparing its intensity to the intensity of voxel $v$, which is expressed by

$$
B_{i}= \begin{cases}0, & \text { if } I_{i}<I_{v} \\ 1, & \text { if } I_{i} \geq I_{v}\end{cases}
$$

where $I_{i}$ denotes the intensity of sample $v_{i}$, and $I_{v}$ denotes the intensity of the center voxel $v$ of $S_{v}$.

Based on (1), the definition of the basic spherical structure pattern (BSSP) is given as follows.

Definition 1: The BSSPs are the binary thresholded spherical surfaces obtained by (1) for each voxel $v \in G$.

The BSSP is monotonic gray-level transformation invariant as monotonic gray-level transformation does not alter the relative intensity differences between the center pixel and the neighboring pixels. Therefore, the binary label assigned to each neighboring pixel remains the same before and after the monotonic gray-level transformation; thus, the BSSP remains the same. Therefore, we have the following property of the BSSP.

Property 1: BSSPs are monotonic gray-level transformation invariant.

Therefore, the BSSP is robust against the monotonic graylevel bias fields, which commonly exist in brain magnetic resonance (MR) images; its occurrence histogram can be served as the signature for each voxel. However, there are many types of BSSPs that can make the histogram of BSSPs too sparse to reliably reflect the anatomical properties of input images. Therefore, only a subset of BSSPs is considered, namely, the USSP, which represents fundamental image structures. The USSP is defined as follows.

Definition 2: The USSPs are BSSPs that have at most two continuous regions of "O"s and "l"s.

For example, Fig. 1(a) is a USSP as it has only one continuous region of "1"s. Fig. 1(b) is also a USSP as it has one continuous region of " 0 "s and one continuous region of "1"s. However, Fig. 1(c) is not a USSP as it has more than two continuous regions of " 0 "s and " 1 "s.

The USSP has some important physical meanings with respect to the fundamental image structures. For example, Fig. 1(a) demonstrates that there is a dark spot at the center voxel. Fig. 1(b) shows that there is an edge along the boundary of the " 0 "s and " 1 "s regions. To further demonstrate that the USSP indeed has dominant proportions among all the BSSPs, Fig. 2 shows the percentages of the USSP among all the BSSPs of the 20 real 3-D image volumes obtained from IBSR ${ }^{1}$ with different values of radius $R$ and numbers of neighboring samples $N$. It is shown that the USSPs of most of the images have over $80 \%$ proportions among all the BSSPs with different values of $R$ and $N$. Therefore, it is reflected that USSPs have dominant proportions among all the BSSPs. Another interesting observation is that, when $R$ is fixed, the proportion of USSPs of each image is similar with different values of $N$. On the other hand, when $N$ is fixed, the proportion of USSPs of each image

\footnotetext{
${ }^{1}$ http://www.cma.mgh.harvard.edu/ibsr/.
} 


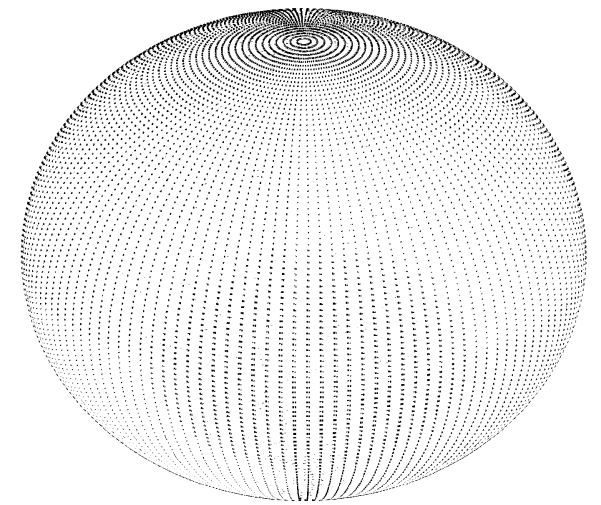

(a)

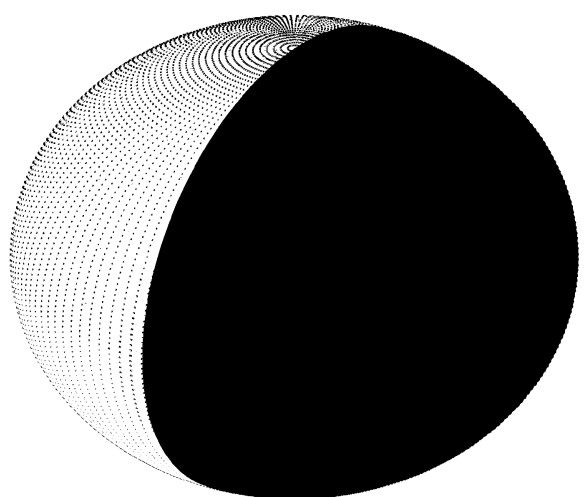

(b)

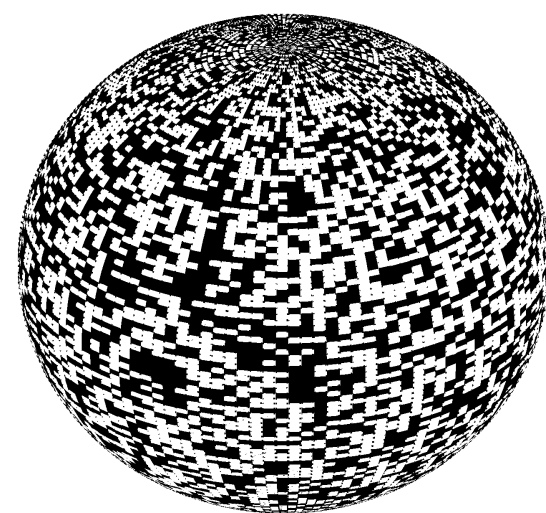

(c)

Fig. 1. (a) Example of a USSP: It has only one continuous region of "1"s. (b) Example of a USSP: It has two continuous regions of "0"s and " 1 "s. (c) Non-USSP: It has more than two continuous regions of " 0 "s and " 1 "s.

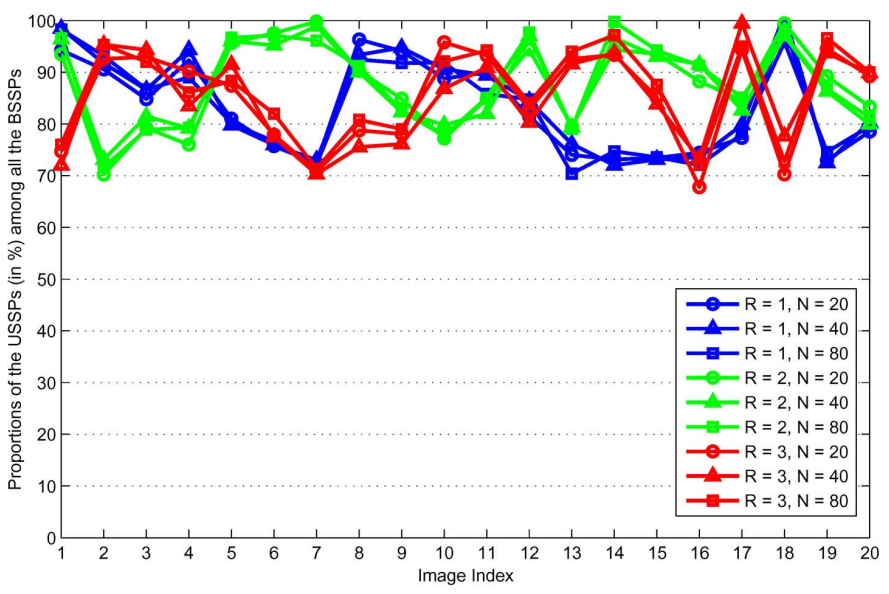

Fig. 2. Proportions of USSPs (in percentage) among all the BSSPs with different values of radius $R$ and number of neighboring samples $N$.

with different values of $R$ varies. The reason is that $R$ controls the scales of interest. Patterns at different scales of interest with respect to the center voxel represent different shapes and structural configurations; thus, the proportions of USSPs vary with respect to $R$. The number of neighboring voxels $N$ represents how accurate is the representation of the shape characterized by the pattern (i.e., how many voxels are sampled from the pattern). As long as the shape remains unchanged, increasing the value of $N$ will not change the uniform property of the pattern if sufficient number of samples is drawn from the pattern. Therefore, it is shown that when $R$ is fixed, the proportion of USSPs of each image is similar with different values of $N$.

Algorithm 1 presents the procedure of determining whether an input BSSP is a USSP or not. The breadth first search (BFS) in Operation 2 takes $O(N)$ time, and Operation 3 also takes $O(N)$ time. Other operations take constant time. Therefore, Algorithm 1 can be completed in $O(N)$ time, which is a linear time process. Since the USSP is a subset of BSSP, it is also monotonic gray-level transformation invariant; thus, we have property 2 below.
Algorithm 1: Determining whether a BSSP is a USSP

Input: A BSSP with radius $R$ and $N$ neighboring voxels on the surface.

Output: true or false (whether the input is a USSP or not).

1.Randomly select a neighboring voxel $i$ on the surface of the input BSSP

2.Find the largest connected component starting from $i$ based on its binary digit $B_{i}$ using the BFS; set a flag for each voxel belonging to that largest connected component

3.FOR each unflagged neighboring voxel $t$ with binary digit $B_{t}$ 4.

$$
\operatorname{IF}\left(B_{t}==B_{i}\right)
$$

5.

Return false

6.

\section{END IF}

\section{END FOR}

\section{Return true}

Property 2: The USSP is monotonic gray-level transformation invariant.

In this paper, the ID of each kind of USSP is determined as the area of the " 0 "s region of the USSP (i.e., the number of the " 0 " digits on the surface of the USSP). All the nonuniform BSSPs are considered as a single type of pattern. Such ID assignment makes the USSP feature rotation invariant because no matter how the image rotates, the area of the "0"s region remains the same. Thus, we have property 3 as follow.

Property 3: The ID assignment of the USSP based on the area of the "O"s region on the surface of the USSP makes the USSP feature rotation invariant.

It should be noted that considering the percentage of the " 0 "s region on the surface of the USSP may be also served as an alternative ID assignment method for the USSP. However, it requires an additional quantization step to convert the percentage value to a specific histogram bin number to construct the USSP histogram. 
After specifying the rotation invariant ID assignment method of the USSP, we can now use the histogram of the USSP as features to represent each voxel. Algorithm 2 describes how to extract rotation and monotonic gray-level transformation invariant USSP features as signatures for each voxel.

Algorithm 2: Calculate the rotation-invariant USSP feature for each voxel

Input: Input image $G$, local cubic square window $W$ for each voxel, USSP radius $R$, and the number of neighboring samples $N$.

Output: Vector image $K$, each voxel is represented by a USSP signature.

1.FOR each voxel $v \in G$

2. $\quad$ SubVolume $=(W$ center at $v)$

3. Initialize a new feature histogram, $H[0 \ldots(N+1)]=0$

4. FOR each voxel $t \in$ SubVolume

5. Calculate its corresponding BSSP $Q_{t}$ with parameters $R$ and $N$

6. Determine whether $Q_{t}$ is a USSP or not using Algorithm 1

7. IF $Q_{t}$ is a USSP

8. PatternID = Number of " 0 "s in $Q_{t}$

9. $\quad H[$ PatternID $]=H[$ PatternID $]+1$

10. ELSE

11. $H[N+1]=H[N+1]+1$

12. END IF

13. END FOR

14. Normalize $H[0 \ldots(N+1)]$ such that $\sum_{i=0}^{N+1} H[i]=1$

15. $K(v)=H[0 \ldots(N+1)]$

\section{END FOR}

\section{Return $K$}

The multiresolution analysis can be achieved in Algorithm 2 by using different values of radius $R$ to calculate the USSP feature histograms and then concatenate all the USSP feature histograms into a single global feature histogram.

\section{B. UGSP}

Here, the UGSP is introduced, which represents the second-order voxel-wise interaction information as the complement with the USSP.

The UGSP considers the gradient orientations of the neighboring voxels. Suppose for input image $G$, for each voxel $v \in G$, spherical neighborhood $S_{v}$ centered at $v$ with radius $R$ is defined. $N_{v}$ samples are taken on the surface of $S_{v}$. Let $\nabla_{v_{i}}$ denote the image gradient of the $i$ th sample $v_{i}\left(i=1, \ldots, N_{v}\right)$ on the surface of $S_{v}$. Then, a rotation-invariant orientation measure $\theta_{v_{i}}$ is defined for each neighboring voxel $v_{i}$, which is the angle between the orientation of $\nabla_{v_{i}}$, and the direction of $v-v_{i} \cdot \theta_{v_{i}}$ can be calculated by

$$
\theta_{v_{i}}=\arccos \frac{\nabla v_{i} \cdot\left(v-v_{i}\right)}{\left|\nabla v_{i}\right| \cdot\left|v-v_{i}\right|}
$$

where $\left|\nabla v_{i}\right|$ and $\left|v-v_{i}\right|$ denote the magnitudes of $\nabla v_{i}$ and $v-v_{i}$, respectively.

The measure of $\theta_{v_{i}}$ is invariant to image rotation because no matter how the images rotate, the relative angle between $\nabla_{v_{i}}$ and the direction of $v-v_{i}$ remains the same.

After calculating $\theta_{v_{i}}$ for each neighboring voxel $v_{i}, \theta_{v_{i}}$ is assigned with a label by uniformly partitioning the angle space into four subspace regions. Such operation can be expressed by

$$
l\left(v_{i}\right)= \begin{cases}1, & \text { if } \theta_{v_{i}} \in\left[0, \frac{\pi}{4}\right) \\ 2, & \text { if } \theta_{v_{i}} \in\left[\frac{\pi}{4}, \frac{\pi}{2}\right) \\ 3, & \text { if } \theta_{v_{i}} \in\left[\frac{\pi}{2}, \frac{3 \pi}{4}\right) \\ 4, & \text { if } \theta_{v_{i}} \in\left[\frac{3 \pi}{4}, \pi\right] .\end{cases}
$$

Then, similar to the definition of BSSP introduced in Section II-A, the basic gradient spherical pattern (BGSP) is defined as follows based on (2) and (3).

Definition 3: The BGSP is the labeled spherical surface obtained from the original spherical neighborhood centered at the reference voxel by using (2) and (3).

The BGSP also has the monotonic gray-level transformation invariant property. As the monotonic gray-level transformation affects the absolute gradient magnitude of each neighboring voxel, the gradient orientation remains the same.

Then, following the same line of the USSP, we also consider a subset of BGSP, namely, the UGSP. Before we define the UGSP, we first define the term "uniform region."

Definition 4: Uniform region is the area on the surface of the $B G S P$ where all the voxels belonging to this area have the same label defined in (3).

Then, the UGSP is defined as follows.

Definition 5: UGSPs are BGSPs whose surfaces can be covered by at most two uniform regions.

It should be also noted that there is a tradeoff if we increase or decrease the number of partitions for the angle space specified in (3). If fewer partitions are considered, then the discriminant power of the gradient spherical pattern may be lower as the division of angle space becomes rougher. However, if the angle space is divided into too many subspace regions, the UGSP feature will be more sensitive to noise, and the histogram of the UGSP features will become more sparse as it is more difficult for a BGSP to be a UGSP when the number of possible labels to represent the angle space is increased. Based on the experimental results, it is found that dividing the angle space into four regions is a good choice given such tradeoff.

Algorithm 3 presents the procedure of determining whether a BGSP is a UGSP or not. It is similar to Algorithm 1, which determines whether a BSSP is a USSP or not, except that in 
Algorithm 3, two neighboring voxels are considered to be connected if and only if they have the same label calculated by (3).

Algorithm 3: Determining whether a BGSP is a UGSP

Input: A BGSP with radius $R$ and $N$ neighboring voxels on the surface.

Output: true or false (whether the input is a UGSP or not).

1.Initialize all the neighboring voxels as unflagged.

2.IF any neighboring voxel in the input BGSP does not have gradient information

\section{Return false}

4.END IF

5.FOR $j=1$ to 2

6. IF all the neighboring voxels are flagged

$7 . \quad$ Break;

8. ELSE

9. Randomly select an unflagged neighboring voxel $v_{i}$ on the surface of the input BGSP.

10. Find the largest connected component $C_{j}$ starting from $v_{i}$ based on its label $l\left(v_{i}\right)$ using the BFS; set a flag for each voxel belonging to that largest connected component. Two neighboring voxels $v_{m}$ and $v_{n}$ are considered to be connected if and only if $l\left(v_{m}\right)==l\left(v_{n}\right)$.

\section{END IF}

\section{END FOR}

13.IF all the neighboring voxels are flagged

14. Return true

15.ELSE

16. Return false

\section{END IF}

The ID assignment method for the UGSP is designed as follows: First, determine whether the input BGSP is a UGSP or not using Algorithm 3. If the input BGSP is a UGSP, then the size of each uniform region $\left|C_{1}\right|$ and $\left|C_{2}\right|$ can be calculated by Algorithm 3. The ID of the UGSP is based on the size of the largest connected component of the UGSP (e.g., $\max \left(\left|C_{1}\right|,\left|C_{2}\right|\right) ; C_{1}$ and $C_{2}$ are calculated by operation 10 in Algorithm 3; and $\left|C_{1}\right|$ and $\left|C_{2}\right|$ denote the sizes of $C_{1}$ and $C_{2}$, respectively).

\section{Combining the USSP and the UGSP as the USRD Feature}

As discussed in the previous sections, the USSP and the UGSP represent the first- and second-order voxel-wise interaction information, respectively. Therefore, intuitively, the USSP and the UGSP can provide complementary information, which motivate us designing the USRD feature. The USRD is the 2-D joint histogram of the USSP and UGSP features.

The procedure of extracting the USRD feature as signatures for each voxel is given in Algorithm 4.

Algorithm 4: Calculate the USRD feature for each voxel

Input: Input image $G$, local cubic square window $W$ for each voxel, radius parameter $R$, and the number of neighboring samples $N$.

Output: Feature image $K$, each voxel is represented by a USRD signature.

1.FOR each voxel $v \in G$

2. $\quad$ SubVolume $=(W$ center at $v)$

3. Initialize a new 2-D feature histogram, $H[0 \ldots(N+1), 0 \ldots(N-\lceil(N / 2)\rceil+1)]=0$

4. FOR each voxel $t \in$ SubVolume

5. Calculate its corresponding $\mathrm{BSSP} \mathrm{BS}_{t}$ with parameters $R$ and $N$; determine whether $\mathrm{BS}_{t}$ is a USSP with Algorithm 1

6. Calculate its corresponding BGSP BG $\mathrm{B}_{t}$ with parameters $R$ and $N$; determine whether $\mathrm{BG}_{t}$ is a UGSP with Algorithm 3

7. $\mathrm{IF} \mathrm{BS}_{t}$ is a USSP

8. $\quad \mathrm{D}_{U S}=$ Number of " 0 "s in $\mathrm{BS}_{t}$

9. ELSE

10. $\operatorname{ID}_{U S}=N+1$

11. END IF

12. $\mathrm{IF} \mathrm{BG}_{t}$ is a UGSP

13. $S=$ Size of the largest connected component in $\mathrm{BG}_{t}$

14. $\quad \mathrm{ID}_{\mathrm{UG}}=S-\lceil(N / 2)\rceil$

15. ELSE

16. $\quad \mathrm{ID}_{\mathrm{UG}}=N-\lceil(N / 2)\rceil+1$

17. END IF

18. $H\left[\mathrm{ID}_{U S}, \mathrm{ID}_{\mathrm{UG}}\right]=H\left[\mathrm{ID}_{U S}, \mathrm{ID}_{\mathrm{UG}}\right]+1$

19. END FOR

20. Normalize $H[0 \ldots(N+1), 0 \ldots(N-\lceil(N / 2)\rceil+1)]$ such that $\sum_{i=0}^{N+1} \sum_{j=0}^{N-\lceil(N / 2)\rceil+1} H[i, j]=1$

21. $K(v)=H[0 \ldots(N+1), 0 \ldots(N-\lceil(N / 2)\rceil+1)]$

22.END FOR

23. Return $K$

The robustness of the USRD against bias field is demonstrated in Fig. 3. Fig. 3(a) is an image slice obtained from 


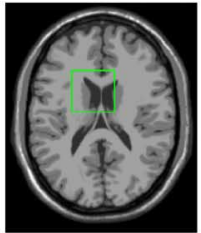

(a)

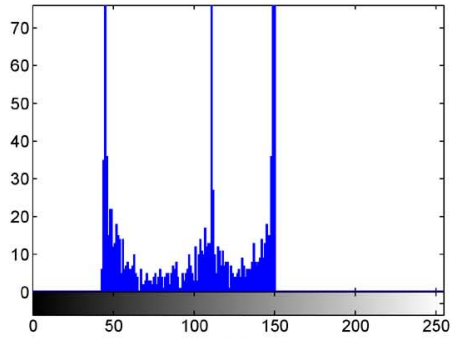

(d)

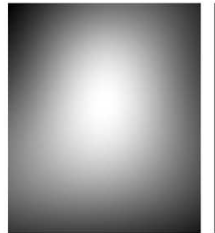

(b)

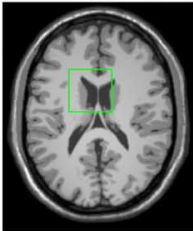

(c)

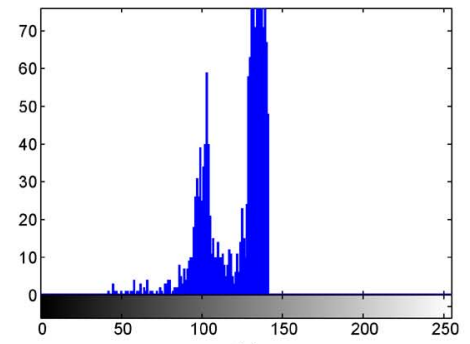

(e)

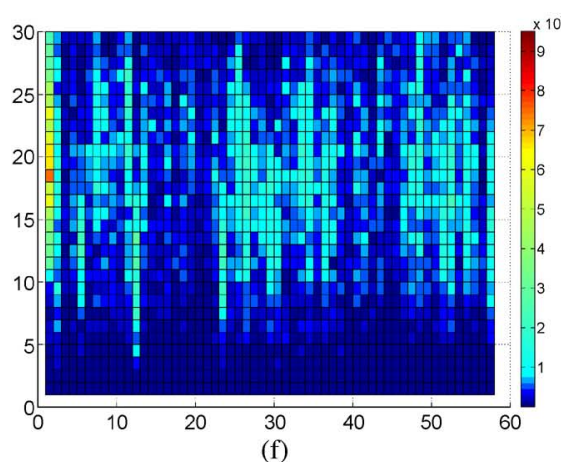

(f)

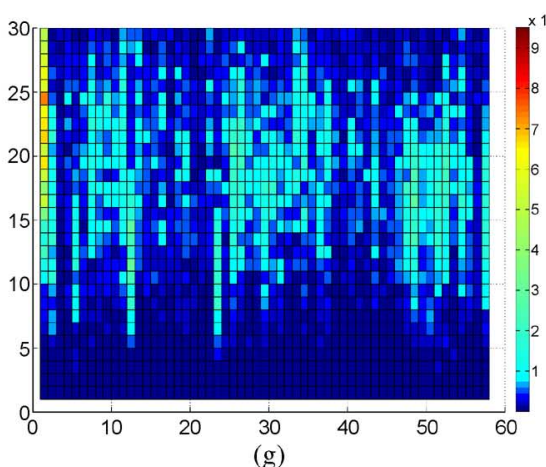

(g)

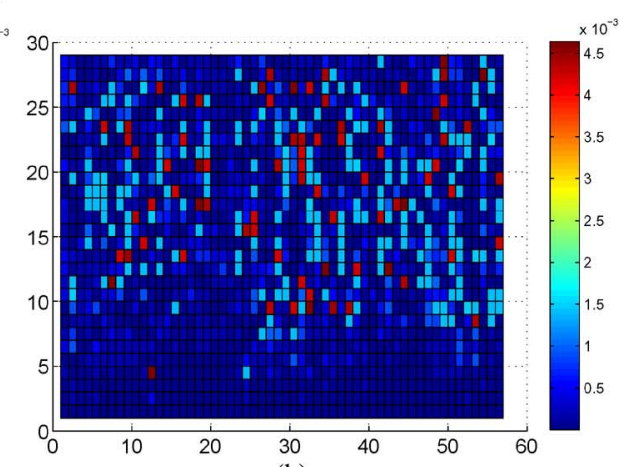

(h)

Fig. 3. (a) Original image without bias field. (b) Type B bias field of $40 \%$ inhomogeneity obtained from BrainWeb. (c) Resulting image after applying the bias field in (b) to the original image in (a). (d) Intensity histogram of [green rectangle region in (a)] ROI in the original image. (e) Intensity histogram of [green rectangle region in (c)] ROI in the bias field distorted image. (f) The USRD feature of ROI in the original image. (g) The USRD feature of ROI in the bias field distorted image. (h) The absolute value of the USRD feature difference of (f) and (g).

BrainWeb2 without any bias field distortion. Fig. 3(b) is the Type B bias field with $40 \%$ inhomogeneity, which is also obtained from BrainWeb. Fig. 3(c) is the resulting image after applying the bias field shown in Fig. 3(b) to the original image in Fig. 3(a). The green rectangle denotes the region of interest (ROI), where features are extracted from it. Fig. 3(d) and (e) are the voxel intensity histograms calculated from the ROI of images in Fig. 3(a) and (c), respectively. Fig. 3(f) and (g) are the USRD features (the 2-D histogram) extracted from the ROI of images in Fig. 3(a) and (c), respectively. It is shown in Fig. 3(d) and (e) that the intensity histograms of ROI before and after bias field distortion are significantly different from each other. For the USRD features, as shown in Fig. 3(f) and (g), it is visually shown that they are very similar to each other before and after bias field distortion, except a slight difference, which can reflect the robustness of the USRD feature. To better visualize the similarity of the USRD feature shown in Fig. 3(f) and (g), we also calculate the absolute values of the USRD feature differences shown in Fig. 3(f) and (g), and the resulting difference map is shown in Fig. 3(h). It is shown in Fig. 3(h) that most of the entries are diminished (i.e., zero or closed to zero), which illustrates the similarity of the USRD

\footnotetext{
${ }^{2}$ http://www.bic.mni.mcgill.ca/brainweb/.
}

feature shown in Fig. 3(f) and (g) before and after the bias field distortion.

The discriminant power of the USRD feature can be also visualized by the feature difference map. Fig. 4(a) is a skullstripped input image obtained from BrainWeb. The voxel highlighted with the green cross denotes the reference voxel whose USRD feature signature is compared with all the other voxels' USRD feature signatures by subtracting the reference voxel's USRD signature to other voxels' USRD signatures and take the L1 norm. Fig. 4(b) is the corresponding color-coded difference map, where blue color denotes high similarity, whereas red color denotes low similarity. To better visualize the similarity map at the ventricle areas where the reference voxel is located, the ROI highlighted by the red square in Fig. 4(b) is zoomed in and shown in Fig. 4(c).

In the next section, the FSC will be adopted to quantitatively compare different types of features.

\section{Feature Discriminant Power Comparison USING THE FSC}

Here, different types of features are directly compared by the Fisher separation protocol with respect to their discriminative power. 


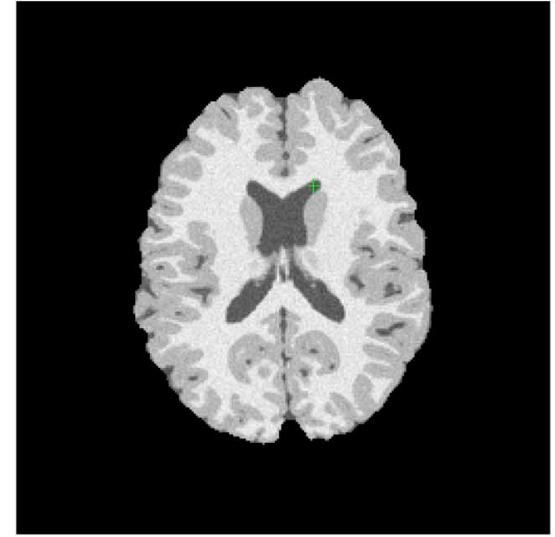

(a)

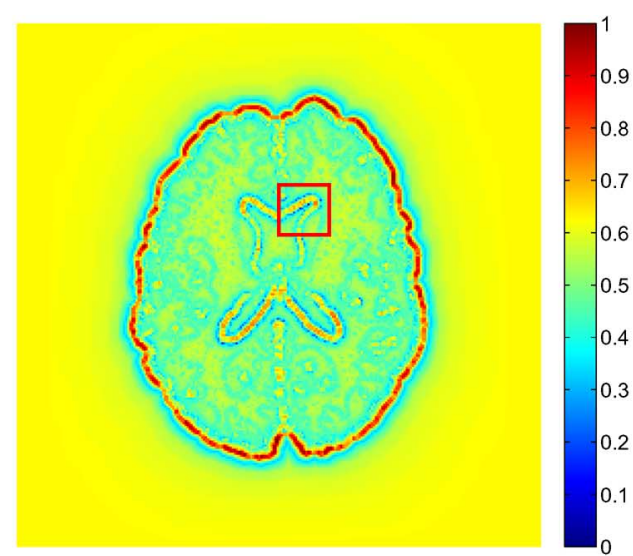

(b)

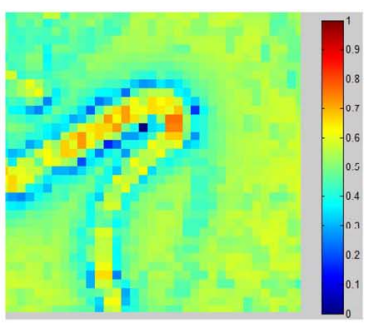

(c)

Fig. 4. (a) Skull-stripped input image obtained from BrainWeb. The reference voxel is highlighted with the green cross. Please refer to the electronic version of the paper to identify the green cross more easily. (b) The color-coded difference map calculated by comparing the USRD feature signature of the reference voxel in (a) with all the other voxels in the input image, where blue color denotes high similarity and red color denotes low similarity. (c) The zoomed-in window of the region of interest highlighted with the red square in (b).

\section{A. Formulation of the Fisher Separation Protocol}

The FSC protocol can be summarized as follows. Let $G$ denote the input image, which is segmented into $c$ classes of tissues. Let $\vec{f}_{i, j}$ denote the feature vector of the $j$ th voxel belonging to the $i$ th tissue and $N_{i}$ denote the number of voxels belonging to the $i$ th tissue. Then, the mean feature vector of each tissue class can be calculated by using the following:

$$
\vec{m}_{i}=\frac{\sum_{j=1}^{N_{i}} \vec{f}_{i, j}}{N_{i}}
$$

where $\vec{m}_{i}$ denotes the mean feature vector of the $i$ th class of tissue.

The FSC aims to find a 1-D projection of the feature vectors in order to maximize the distance between two clusters of different classes. Such projection can be expressed by the following [25]:

$$
k_{z}=\left(\vec{m}_{1}-\vec{m}_{2}\right)^{T} \mathbf{S}^{-1} \vec{f}_{z}
$$

where $\vec{m}_{1}$ and $\vec{m}_{2}$ are the mean feature vectors of two different classes obtained by (4). $\mathbf{S}^{-1}$ is the inverse of the pooled covariance matrix. The pooled covariance matrix is defined by (6), where $U_{i}$ denotes the covariance matrix for class $i . \vec{f}_{z}$ is the feature vector of voxel $z$, where $z$ belongs to tissue classes 1 or 2. $k_{z}$ is the projected value of $\vec{f}_{z}$ onto the 1-D space using the FSC. The projection expressed by (5) maximizes the FSC function defined by (7), i.e.,

$$
\begin{aligned}
& \mathbf{S}=\left(\frac{1}{\sum_{i=1}^{c} N_{i}-1}\right)\left(\sum_{i=1}^{c} \frac{U_{i}}{N_{i}-1}\right) \\
& f=\frac{\left|C_{1}-C_{2}\right|}{\sqrt{\sigma_{1}^{2}+\sigma_{2}^{2}}}
\end{aligned}
$$

where $\sigma_{1}^{2}$ and $\sigma_{2}^{2}$ denote the variances of the projected feature vectors of classes 1 and 2, respectively. $C_{1}$ and $C_{2}$ are the projected mean feature vectors of classes 1 and 2, which are calculated by

$$
C_{i}=\left(\vec{m}_{1}-\vec{m}_{2}\right)^{T} \mathbf{S}^{-1} \vec{m}_{i} \quad(i=1,2) .
$$

The larger the value of (7), the higher the discriminant power the feature has.

In this paper, five types of features are compared. They are as follows: 1) voxel intensity; 2) the GMIs feature used in HAMMER [14]; 3) the LHF used in the extension of HAMMER [24]; 4) the symmetric Alpha stable $(S \alpha S)$ filtered-based features in [25]; and 5) the proposed USRD feature. In order to observe the performance of each component of the USRD, we also evaluate the USSP and UGSP features individually. For clarity purpose, we briefly describe the four different features for comparison in the following sections.

\section{B. GMI Feature}

The GMI feature is derived based on the 3-D regular moments defined in

$$
M_{p, q, r}=\underset{\left(x_{1}\right)^{2}+\left(x_{2}\right)^{2}+\left(x_{3}\right)^{2}<R^{2}}{\int} x_{1}^{p} x_{2}^{q} x_{3}^{r} f_{\text {tissue }}\left(x_{1}, x_{2}, x_{3}\right)
$$

where $\left(x_{1}, x_{2}, x_{3}\right)$ denote the 3 -D coordinates of the voxel under consideration. It is also assumed that the center of the coordinate system has been shifted to $\left(x_{1}, x_{2}, x_{3}\right)$ in (9). The order of the 3-D regular moment is determined by $(p+q+r)$. The integration is defined within a sphere with radius $R$, where $R$ denotes the scale of interest. $f_{\text {tissue }}\left(x_{1}, x_{2}, x_{3}\right)$ denotes the membership function for each tissue (i.e., gray matter (GM), white matter (WM), and cerebrospinal fluid (CSF) in this paper). GMIs feature with different orders can be derived from the 3 -D regular moments.

\section{LHFs}

The LHF is proposed in the extension of HAMMER [24], which does not require the segmentation results. For each voxel $x$ of the input image, a spherical neighborhood with radius $R$ is first defined. Then, the intensity histogram $h(x)$ inside this spherical neighborhood is calculated. After obtaining $h(x)$, the regular geometric moment is calculated as follows:

$$
m(x, p)=\sum_{i} i^{p} h(x, i)
$$


TABLE I

MEAn VAlues of the FSC FunCtion IN (7) FOR DifFERENT KINDS OF TISSUE PAIRS: GM AND WM, GM AND THE CSF, AND WM AND THE CSF OF VARIOUS Features of the 20 Image Volumes ObTained From BrainWeb Without ANy Bias Field Distortion

\begin{tabular}{|c|c|c|c|c|c|c|c|}
\hline Features & Intensity & GMIs & LHFs & $S \alpha S+$ MRO & USSP & UGSP & USRD \\
\hline Gray \& White & 3.27 & 10.86 & 7.82 & 13.59 & 13.22 & 11.03 & 15.17 \\
Gray \& CSF & 3.44 & 13.79 & 10.15 & 15.64 & 13.86 & 14.26 & 16.03 \\
White \& CSF & 3.08 & 14.10 & 11.23 & 16.07 & 15.01 & 14.60 & 16.84 \\
\hline
\end{tabular}

TABLE II

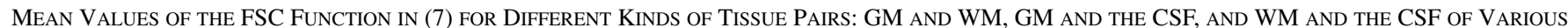

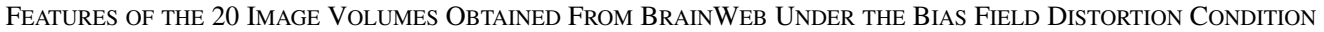

\begin{tabular}{|c|c|c|c|c|c|c|c|}
\hline Features & Intensity & GMIs & LHFs & $S \alpha S+$ MRO & USSP & UGSP & USRD \\
\hline Gray \& White & 1.28 & 7.72 & 4.85 & 9.74 & 11.04 & 10.30 & 13.73 \\
Gray \& CSF & 2.15 & 8.03 & 6.02 & 10.26 & 12.16 & 12.74 & 14.26 \\
White \& CSF & 1.83 & 8.96 & 5.68 & 9.42 & 13.63 & 12.41 & 14.68 \\
\hline
\end{tabular}

where $p$ is an integer denoting the order of the regular geometric moment, and $h(x, i)$ denotes the occurrence frequency of intensity $i$ in histogram $h(x)$. In LHF [24], $p$ ranges from 0 to 2. Following the same setting in [24], the regular moment is combined with the response value of the Canny edge detector to serve as the voxel feature signature.

\section{Symmetric Alpha-Stable Filtered-Based Features}

The symmetric Alpha stable $(S \alpha S)$ features are extracted by passing the input images through the $S \alpha S$ filter banks [25]. The 3-D $S \alpha S$ filter can be expressed by [25]

$\psi_{\alpha, \gamma, \tau, \theta, \phi}(x, y, z)=f_{\alpha, \gamma}(x, y, z) \cdot \exp (j 2 \pi(x u+y v+z w))$

where $f_{\alpha, \gamma}(x, y, z)$ is the zero-mean $S \alpha S$ kernel defined by

$$
f_{\alpha, \gamma}(x, y, z)=\left(\frac{1}{\sqrt{2 \pi}}\right)^{3} \int_{u} \int_{v} \int_{w} A \cdot B d w d v d u
$$

where $A$ and $B$ are defined by (13) and (14), respectively, as

$$
\begin{aligned}
& A=\exp \left(-\gamma\left(x^{2}+y^{2}+z^{2}\right)^{\frac{\alpha}{2}}\right) . \\
& B=\exp (j(x u+y v+z w)) .
\end{aligned}
$$

In (11), $\alpha$ is the coefficient controlling the heaviness of the tail of $S \alpha S$ kernels. $\gamma$ is the scale parameter similar to the variance of the Gaussian kernel. $(u, v, w)$ denote the 3-D frequency components in the Fourier domain. $\tau=\sqrt{u^{2}+v^{2}+w^{2}}$ is the center frequency. $\theta$ and $\phi$ together represent the orientation in the 3-D frequency domain, where $0 \leq \phi \leq \pi$ and $0 \leq \theta \leq \pi$. $(u, v, w)$ satisfy the following: 1) $u=\tau \sin \phi \cos \theta$;2) $v=\tau \sin \phi \sin \theta$; and 3) $w=\tau \cos \phi$. In this paper, the parameter settings of the $S \alpha S$ filters follow the same setting in [25].

\section{E. Feature Discriminant Power Comparison With the FSC}

Here, we first test the basic discriminant power of different kinds of features. Table I lists the FSC function's values of each pair of tissues obtained by using different types of features of the 20 image volumes obtained from BrainWeb, without any bias field distortion. Three radii values of the USRD were used to achieve the multiresolution analysis, i.e., $R_{1}=1, R_{2}=3$, and $R_{3}=6$, with the number of neighboring samples $N=$ 80 for each radius. Obviously, the simplest feature is the voxel intensity alone, and therefore, it can be served as the baseline.
It is shown in Table I that all the features outperform the baseline (i.e., using intensity alone) because they have larger FSC value, which means that they have higher discriminant power than using intensity alone. The GMIs feature used in HAMMER [14] has higher discriminant power than the LHF feature used in the extension of HAMMER [24] as the GMIs include the prior segmentation information of input images. The $S \alpha S$ feature [25] has even higher discriminant power than the GMIs feature. If the two components of the USRD (i.e., USSP and UGSP) are individually used, they are comparable to the $S \alpha S+$ MRO feature. If the complete USRD feature is used, it has the highest discriminant power among all the compared features. Therefore, the complementary property of the USSP and the UGSP is also implied.

Then, we evaluate the robustness of different kinds of features against bias fields. There are three types of bias fields provided by BrainWeb. Each image is applied with a bias field by the following procedure: First, the type of the bias field is determined by randomly selecting from the three types of bias fields; then, the image volume is applied with the selected bias fields with $40 \%$ inhomogeneity (intensity nonuniformity). Table II lists the FSC values of different kinds of features under the bias field distortion condition.

It is shown in Table II that the FSC values of different kinds of features for each pair of tissues drop, as compared with Table I, due to the bias field distortion. The robustness of the proposed feature is reflected in Table II, and even for the USSP and UGSP features, they individually outperform all the other compared features. When the USSP and the UGSP are combined to form the USRD feature, the discriminant power is even higher.

\section{MRF LABELING FRAMEWORK AS THE DEFORMATION MODEL}

Here, we briefly describe the MRF labeling framework, which is adopted as the deformation model in this paper.

The fundamental form of the MRF energy function can be expressed by

$$
\begin{aligned}
E_{f} & =E_{\text {data }}+E_{\text {smoothness }} \\
& =\sum_{p \in \Omega} D_{p}\left(l_{p}\right)+\sum_{(p, q) \in N} V_{p, q}\left(l_{p}, l_{q}\right)
\end{aligned}
$$

where $E_{\text {data }}$ is the data term and $E_{\text {smoothness }}$ is the smoothness term. $\Omega$ denotes the set of voxels, and $N$ is the neighborhood 
system used in $\Omega$. The six-connected neighborhood system is used in this paper. $D_{p}\left(l_{p}\right)$ is the energy function associated with the data term, and $V_{p, q}\left(l_{p}, l_{q}\right)$ is the energy function associated with the smoothness term.

By quantizing the deformation space, the registration problem can be transformed to the MRF labeling problem. A discrete set of labels $L \in\left\{l^{1}, l^{2}, \ldots, l^{n}\right\}$ is defined. Each label $l^{i}(1 \leq i \leq n)$ corresponds to displacement vector $\vec{d}_{i}$. Assigning voxel $p$ with label $l_{p}$ corresponds to moving $p$ to a new position by displacement vector $\overrightarrow{d_{p}}$. The quantization step proposed in [28] is adopted, where each voxel can be displaced off the original position bounded by a discretized window $\Psi=\{0, \pm s, \pm 2 s, \ldots, \pm w s\}^{d}$ of dimension $d$, in this paper $s=1$.

The energy function $D_{p}\left(l_{p}\right)$ associated with the data term is defined based on the USRD features as

$$
\begin{aligned}
D_{p}\left(l_{p}\right) & =D_{p}\left(G_{\text {template }}(\vec{p}), G_{\text {subject }}\left(\vec{p}+\overrightarrow{d_{l_{p}}}\right)\right. \\
& =D_{p}\left(K_{\text {template }}(\vec{p}), K_{\text {subject }}\left(\vec{p}+\overrightarrow{d_{l_{p}}}\right)\right) \\
& =\operatorname{JSD}\left(K_{\text {template }}(\vec{p}) \| K_{\text {subject }}\left(\vec{p}+\overrightarrow{d_{l_{p}}}\right)\right)
\end{aligned}
$$

where $G_{\text {template }}$ is the template image, $G_{\text {subject }}$ is the subject image, and $K_{\text {template }}$ and $K_{\text {subject }}$ are the USRD feature images of $G_{\text {template }}$ and $G_{\text {subject }}$, respectively, at the current iteration calculated by Algorithm 4. JSD $(\cdot)$ denotes the Jensen-Shannon divergence measure [29] defined by [29]

$$
\operatorname{JSD}(P \| Q)=\frac{1}{2} D(P \| M)+\frac{1}{2} D(Q \| M)
$$

where $P$ and $Q$ are two probability distributions with $M=$ $(1 / 2)(P+Q)$ and $D(\cdot)$ is the Kullback-Leibler divergence function.

The smoothness energy function $V_{p, q}\left(l_{p}, l_{q}\right)$ is defined by the piecewise truncated absolute distance as

$$
V_{p, q}\left(l_{p}, l_{q}\right)=\min \left(\lambda,\left|\overrightarrow{d_{l_{p}}}-\overrightarrow{d_{l_{q}}}\right|\right)
$$

where $\lambda$ is a constant represents the maximum penalty.

The smoothness term defined in (18) enforces the smoothness constraint on the deformation field to avoid the folding and crossing effects that do not exist in realistic deformation fields, which plays a similar role to diffeomorphic transformations [30], [31]. A typical deformation field generated by the proposed method is given in Fig. 5; it is shown that the deformation field is smooth, and no folding occurs.

The $\alpha$-expansion algorithm [32] is applied to minimize the energy function defined in (15).

\section{EXPERIMENTAL RESULTS}

Here, we evaluate the proposed method by performing nonrigid image registration experiments on both the simulated database obtained from BrainWeb and the real 3-D database obtained from IBSR. The proposed method is also compared

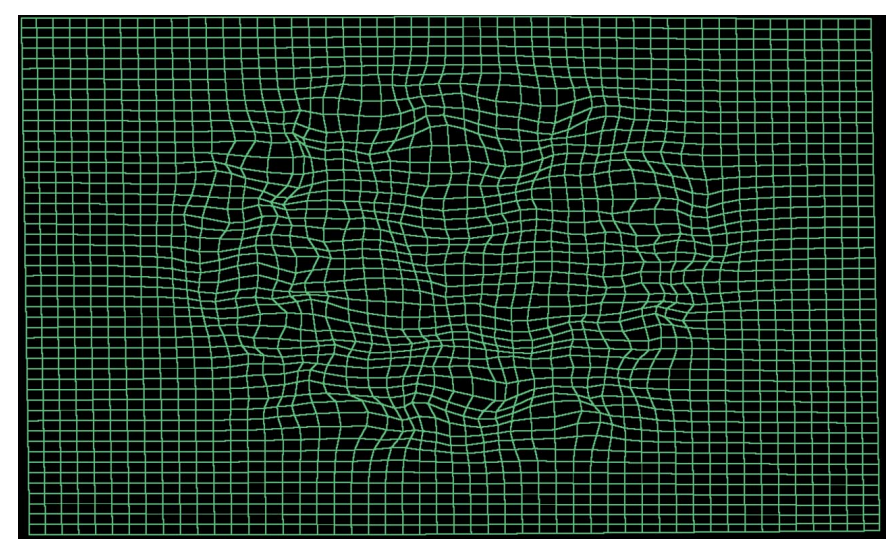

Fig. 5. Typical deformation field obtained by using the proposed method. It is shown that the generated deformation field is smooth and does not have folding effects, which preserves the topology of the anatomical structures.

with five state-of-the-art registration algorithms. They are as follows: 1) the FFD algorithm [22]; 2) Demons algorithm [23]; 3) HAMMER [14]; 4) the extension of HAMMER [24] based on LHF; and 5) the symmetric Alpha-stable $(S \alpha S)$ filtered-based method [25].

In all the experiments, subvolume window size $W$ was set to $16 \times 16 \times 16$, and the number of neighboring samples $N$ was set to 80 in Algorithm 4. Three radii values of the USRD were used to achieve the multiresolution analysis, i.e., $R_{1}=1, R_{2}=3$, and $R_{3}=6$. The 3-D displacement window used in this paper was $\Psi=\{0, \pm 1, \pm 2, \ldots, \pm 12\}^{3}$. The maximum penalty parameter $\lambda$ defined in (18) was set to 15 . Moreover, affine registrations were first applied to register the subject images to the template image before conducting the nonrigid image registration. All the experiments were run on a $3-\mathrm{GHz}$ duo core central processing unit 2-GB random access memory computer.

\section{A. Experiments With Simulated Database}

Here, the proposed method is evaluated on the simulated 3-D brain database from BrainWeb. Twenty image volumes were obtained from BrainWeb, with size $256 \times 256 \times 181$ voxels for each volume. The segmentation result of each image is also provided by BrainWeb; each image is segmented into three classes of tissues (i.e., GM, WM, and CSF). One of the images was used as the template image.

Before performing registration, the skull of each input image was removed as it is a required step for some of the methods compared in this paper, such as HAMMER [14] and the extension of HAMMER [24]. The skull-stripping process was accomplished by using the software Brainsuite version 2 obtained from $\mathrm{USC}^{3}$.

Fig. 6 shows the resulting reconstructed average brain images obtained via different approaches. For comparison purpose, the template image is also shown in Fig. 6(a). It is shown that the average brain images obtained via the proposed method preserve most of the details of the template image, particularly in the ventricular and cortical regions. In Fig. 6, significant differences are highlighted with green circles.

\footnotetext{
${ }^{3} \mathrm{http} / / /$ brainsuite.usc.edu/.
} 


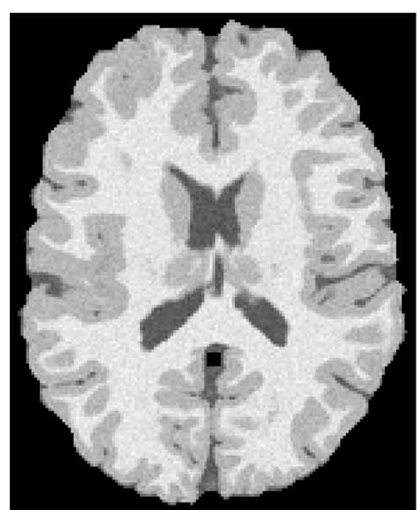

(a)

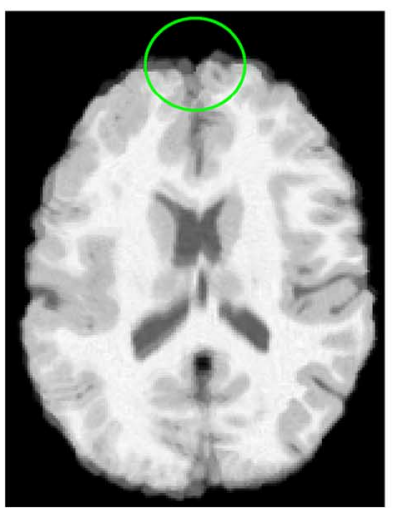

(e)

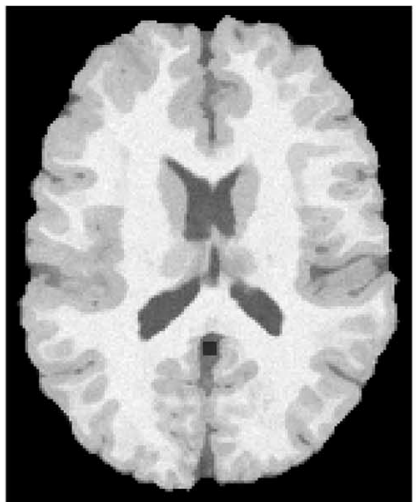

(b)

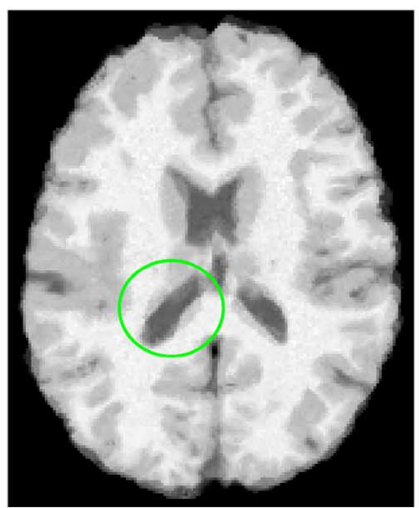

(f)

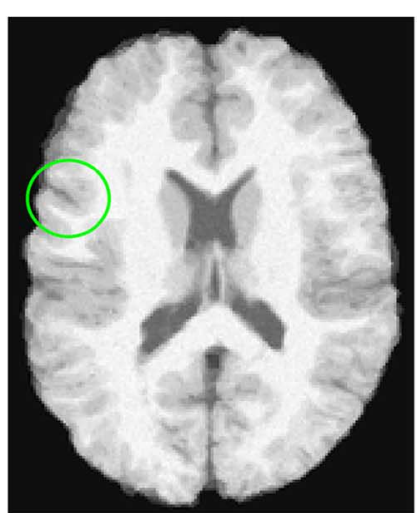

(c)

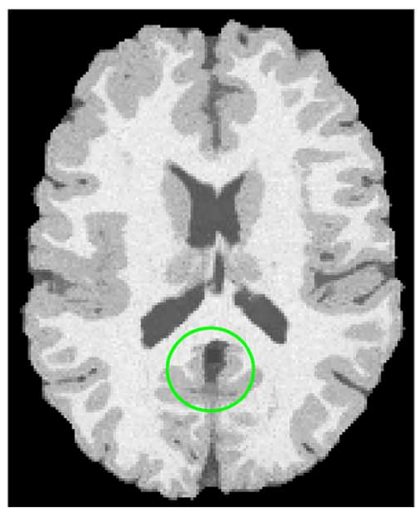

(g)

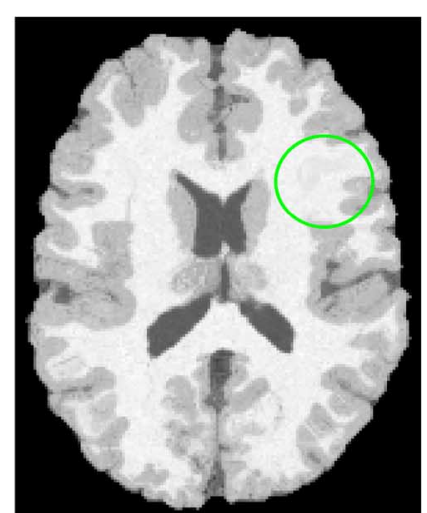

(d)

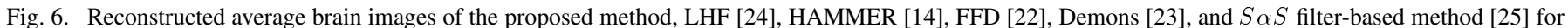

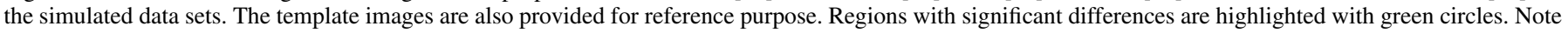
that the skulls have been removed in the preprocessing step. (a) Template. (b) USRD. (c) LHF. (d) HAMMER. (e) FFD. (f) Demons. (g) $S \alpha S$.

TABLE III

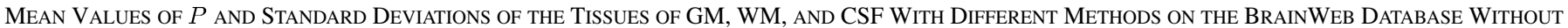
Any Bias Field Distortion. BR Denotes Before Registration. The Highest VAlues of $P$ ARE Bolded FOR EACH Tissue

\begin{tabular}{|c|c|c|c|c|c|c|c|c|c|}
\hline Tissue & BR & FFD & Demons & HAMMER & LHF & $S \alpha S$ & USSP & UGSP & USRD \\
\hline Gray & $0.462 \pm 0.02$ & $0.739 \pm 0.08$ & $0.774 \pm 0.06$ & $0.814 \pm 0.03$ & $0.794 \pm 0.02$ & $0.834 \pm 0.04$ & $0.831 \pm 0.05$ & $0.823 \pm 0.02$ & $\mathbf{0 . 8 6 2} \pm \mathbf{0 . 0 3}$ \\
White & $0.436 \pm 0.04$ & $0.762 \pm 0.04$ & $0.759 \pm 0.04$ & $0.828 \pm 0.04$ & $0.805 \pm 0.02$ & $0.847 \pm 0.03$ & $0.833 \pm 0.03$ & $0.826 \pm 0.04$ & $\mathbf{0 . 8 5 6} \pm \mathbf{0 . 0 2}$ \\
CSF & $0.383 \pm 0.05$ & $0.735 \pm 0.04$ & $0.763 \pm 0.05$ & $0.807 \pm 0.03$ & $0.806 \pm 0.01$ & $0.840 \pm 0.03$ & $0.824 \pm 0.04$ & $0.815 \pm 0.02$ & $\mathbf{0 . 8 6 4} \pm \mathbf{0 . 0 3}$ \\
\hline
\end{tabular}

TABLE IV

Mean Values of $P$ And Standard Deviations of the Tissues of GM, WM, and CSF With DifFerent Methods on the BrainWeb Database Under The Bias Field Distortion Condition. BR Denotes Before Registration. The Highest Values of $P$ ARE Bolded For EACH Tissue

\begin{tabular}{|c|c|c|c|c|c|c|c|c|c|}
\hline Tissue & BR & FFD & Demons & HAMMER & LHF & S $S$ S & USSP & UGSP & USRD \\
\hline Gray & $0.462 \pm 0.02$ & $0.685 \pm 0.06$ & $0.716 \pm 0.05$ & $0.754 \pm 0.03$ & $0.748 \pm 0.04$ & $0.792 \pm 0.03$ & $0.808 \pm 0.04$ & $0.798 \pm 0.04$ & $\mathbf{0 . 8 2 4} \pm \mathbf{0 . 0 4}$ \\
White & $0.436 \pm 0.04$ & $0.714 \pm 0.03$ & $0.731 \pm 0.03$ & $0.772 \pm 0.05$ & $0.760 \pm 0.03$ & $0.792 \pm 0.05$ & $0.814 \pm 0.02$ & $0.803 \pm 0.05$ & $\mathbf{0 . 8 3 7} \pm \mathbf{0 . 0 3}$ \\
CSF & $0.383 \pm 0.05$ & $0.693 \pm 0.05$ & $0.720 \pm 0.02$ & $0.761 \pm 0.02$ & $0.755 \pm 0.03$ & $0.807 \pm 0.05$ & $0.816 \pm 0.03$ & $0.811 \pm 0.03$ & $\mathbf{0 . 8 3 2} \pm \mathbf{0 . 0 5}$ \\
\hline
\end{tabular}

In order to quantitatively evaluate the registration accuracy, we also adopt the tissue overlapping measure function proposed in [33] as the evaluation function. It is defined by

$$
P=\frac{N(A \cap B)}{N(A \cup B)}
$$

where $A$ and $B$ denote the regions of a specific type of tissue of the template and warped subject images. Of course, more sophisticated measure functions can be also used such as the function proposed in [34].

Table III lists the values of $P$ in (19) for different approaches. To investigate the individual performance and complementary properties of the two components of the USRD, i.e., USSP and UGSP, the values of $P$ by individually using the USSP and the UGSP are also reported.
It is shown in Table III that the proposed method has the largest value of $P$ among all the compared methods. Additionally, the final registration results of different approaches matched with the findings in Section III via the FSC protocol (FFD and Demons are both intensity-based approaches).

We also calculated the registration accuracy of different methods under the bias field distortion condition. The bias field distorted images were obtained similar to Section III. The values of $P$ in (19) for different approaches under the bias field distortion condition are listed in Table IV.

It is shown in Table IV that under the bias field distortion condition, the proposed method still maintains the highest registration accuracy among all the compared methods. Therefore, the registration accuracy and robustness of the proposed method are strongly implied. 
TABLE V

Mean Values of $P$ and Standard Deviations of the Tissues of GM, WM, and CSF With Different Methods on the BrainWeb Database After ApPlying the Intensity Standardization Process on the Bias Field Distorted Images. BR Denotes Before Registration. The Highest VAlues of $P$ ARe Bolded For EACh Tissue

\begin{tabular}{|c|c|c|c|c|c|c|c|c|c|}
\hline Tissue & BR & FFD & Demons & HAMMER & LHF & $S \alpha S$ & USSP & UGSP & USRD \\
\hline Gray & $0.462 \pm 0.02$ & $0.702 \pm 0.03$ & $0.748 \pm 0.07$ & $0.796 \pm 0.05$ & $0.761 \pm 0.02$ & $0.805 \pm 0.08$ & $0.812 \pm 0.04$ & $0.810 \pm 0.03$ & $\mathbf{0 . 8 4 6} \pm \mathbf{0 . 0 5}$ \\
White & $0.436 \pm 0.04$ & $0.737 \pm 0.06$ & $0.744 \pm 0.02$ & $0.794 \pm 0.03$ & $0.772 \pm 0.08$ & $0.803 \pm 0.01$ & $0.823 \pm 0.04$ & $0.817 \pm 0.06$ & $\mathbf{0 . 8 4 2} \pm \mathbf{0 . 0 4}$ \\
CSF & $0.383 \pm 0.05$ & $0.718 \pm 0.03$ & $0.745 \pm 0.07$ & $0.782 \pm 0.04$ & $0.779 \pm 0.06$ & $0.818 \pm 0.03$ & $0.819 \pm 0.05$ & $0.813 \pm 0.01$ & $\mathbf{0 . 8 4 1} \pm \mathbf{0 . 0 3}$ \\
\hline
\end{tabular}

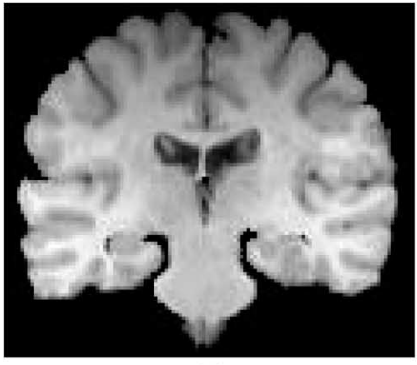

(a)

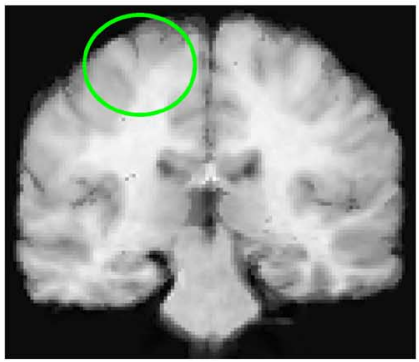

(e)

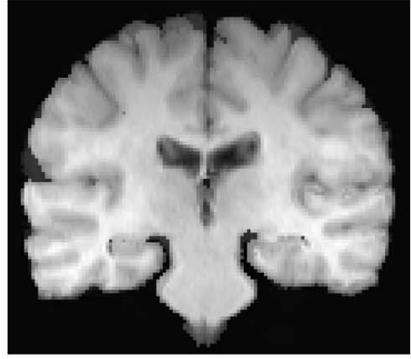

(b)

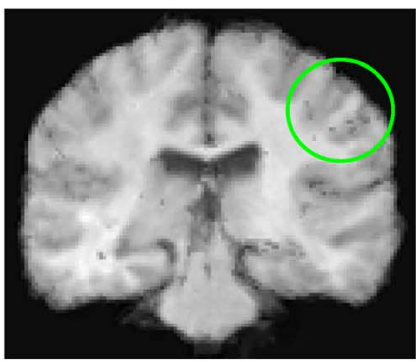

(f)

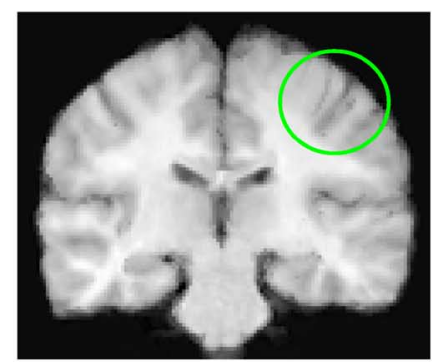

(c)

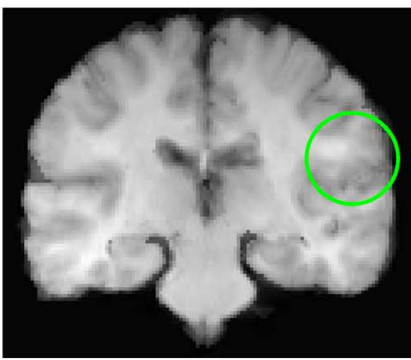

(g)

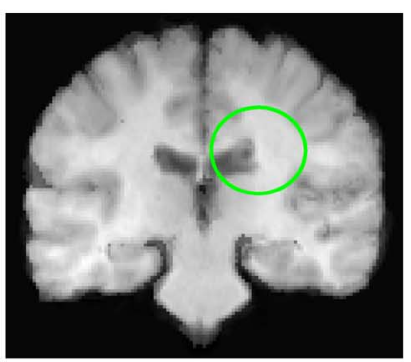

(d)

Fig. 7. Reconstructed average brain images of the proposed method, LHF [24], HAMMER [14], FFD [22], Demons [23], and $S \alpha S$ filter-based method [25] for the real 3-D data sets. The template images are also provided for reference purpose. Regions with significant differences are highlighted with green circles. (a) Template. (b) USRD. (c) LHF. (d) HAMMER. (e) FFD. (f) Demons. (g) $S \alpha S$.

TABLE VI

Mean Values of $P$ and Standard Deviations of the GM, WM, and CSF For Different Methods on the IBSR Database. BR DenOtes Before Registration. The Highest VAlues of $P$ ARe Bolded for Each Tissue

\begin{tabular}{|c|c|c|c|c|c|c|c|c|c|}
\hline Tissue & BR & FFD & Demons & HAMMER & LHF & $S \alpha S$ & USSP & UGSP & USRD \\
\hline Gray & $0.522 \pm 0.04$ & $0.746 \pm 0.03$ & $0.776 \pm 0.04$ & $0.806 \pm 0.04$ & $0.781 \pm 0.02$ & $0.830 \pm 0.02$ & $0.827 \pm 0.06$ & $0.823 \pm 0.04$ & $\mathbf{0 . 8 4 5} \pm \mathbf{0 . 0 3}$ \\
White & $0.553 \pm 0.02$ & $0.761 \pm 0.04$ & $0.779 \pm 0.05$ & $0.787 \pm 0.03$ & $0.783 \pm 0.01$ & $0.842 \pm 0.03$ & $0.835 \pm 0.03$ & $0.846 \pm 0.05$ & $\mathbf{0 . 8 6 0} \pm \mathbf{0 . 0 2}$ \\
CSF & $0.318 \pm 0.03$ & $0.755 \pm 0.04$ & $0.780 \pm 0.04$ & $0.785 \pm 0.04$ & $0.784 \pm 0.03$ & $0.836 \pm 0.01$ & $0.828 \pm 0.04$ & $0.832 \pm 0.05$ & $\mathbf{0 . 8 5 1} \pm \mathbf{0 . 0 4}$ \\
\hline
\end{tabular}

Moreover, in recent years, it is found that intensity standardization as a preprocessing step also plays an important role to help establish more reliable anatomical correspondence [13], [35]. In this paper, we also study the registration performance of different approaches after performing the intensity standardization step. The values of $P$ in (19) for different approaches after the intensity standardization process are listed in Table V.

It is shown in Table $\mathrm{V}$ that the tissue overlapping ratios of different approaches are generally higher after performing the intensity standardization procedure, which reflects the effectiveness of the intensity standardization to help establish more reliable anatomical correspondence, as stated in [13]. It is also shown that the tissue overlapping ratios obtained by the proposed method still maintain the highest among all the compared methods. Thus, the ability to establish more reliable anatomical correspondences of the proposed method is strongly implied.

The average computation time for the proposed method to register one pair of input images for the BrainWeb database was around $3.7 \mathrm{~h}$. The average computation time to register one pair of input images of all the compared methods is also listed as follows: 1) FFD took $4.8 \mathrm{~h}$; 2) Demons took $2.6 \mathrm{~h}$; 3 ) LHF took $5.1 \mathrm{~h}$; and 4) HAMMER took $5.4 \mathrm{~h}$.

\section{B. Experiments With Real Database}

Here, we evaluate the proposed method on the real 3-D database from IBSR. Twenty skull-stripped T1-weighted normal subjects were obtained. Each subject is with resolution $256 \times 256 \times 60$, and the segmentation results of each subject are also provided by IBSR. Each subject is segmented into three classes of tissues (i.e., GM, WM, and the CSF).

Similar to Section V-A, one of the images was used as the template; others served as the subject images. Fig. 7 shows the reconstructed average brain images obtained by different approaches. For comparison purpose, the template image is also shown in Fig. 7(a). It is visually shown that the resulting average brain image obtained via the proposed method is most similar to the template image. Therefore, the registration accuracy of the proposed method is reflected.

The statistical measure function expressed by (19) was also adopted to quantitatively evaluate different approaches. Table VI lists the value of $P$ for different methods for the real 3-D database obtained from IBSR.

It is shown in Table VI that the proposed method still maintains the highest registration accuracy among all the compared methods as it has the largest value of $P$. 
TABLE VII

Mean Values of $P$ and Standard Deviations of the GM, WM, AND CSF For DifFerent Methods on the IBSR Database After Applying the INTENSITY STANDARDIZATION PROCESS. BR DENOTES BEFORE REgISTRATION. THE HigheSt VALUES OF $P$ ARE BOLDED FOR EACH TISSUE

\begin{tabular}{|c|c|c|c|c|c|c|c|c|c|}
\hline Tissue & BR & FFD & Demons & HAMMER & LHF & $S \alpha S$ & USSP & UGSP & USRD \\
\hline Gray & $0.522 \pm 0.04$ & $0.764 \pm 0.04$ & $0.795 \pm 0.06$ & $0.819 \pm 0.03$ & $0.797 \pm 0.04$ & $0.836 \pm 0.08$ & $0.832 \pm 0.04$ & $0.828 \pm 0.03$ & $\mathbf{0 . 8 4 9} \pm \mathbf{0 . 0 2}$ \\
White & $0.553 \pm 0.02$ & $0.783 \pm 0.07$ & $0.786 \pm 0.02$ & $0.798 \pm 0.05$ & $0.791 \pm 0.06$ & $0.845 \pm 0.04$ & $0.840 \pm 0.01$ & $0.848 \pm 0.04$ & $\mathbf{0 . 8 6 2} \pm \mathbf{0 . 0 4}$ \\
CSF & $0.318 \pm 0.03$ & $0.762 \pm 0.05$ & $0.794 \pm 0.02$ & $0.806 \pm 0.07$ & $0.790 \pm 0.04$ & $0.838 \pm 0.03$ & $0.831 \pm 0.02$ & $0.835 \pm 0.03$ & $\mathbf{0 . 8 5 4} \pm \mathbf{0 . 0 2}$ \\
\hline
\end{tabular}

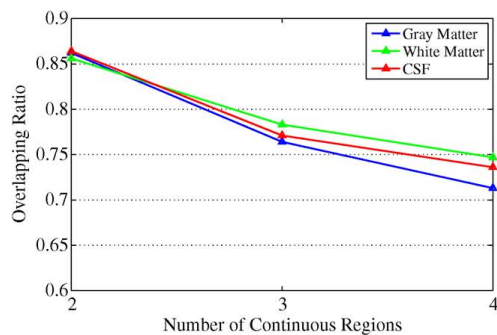

(a)

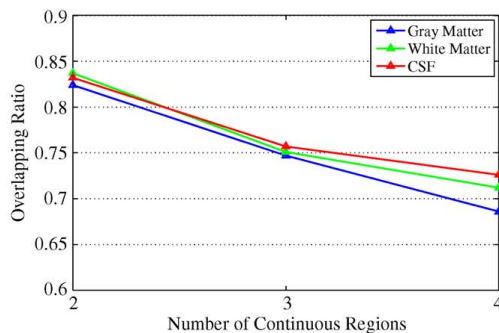

(b)

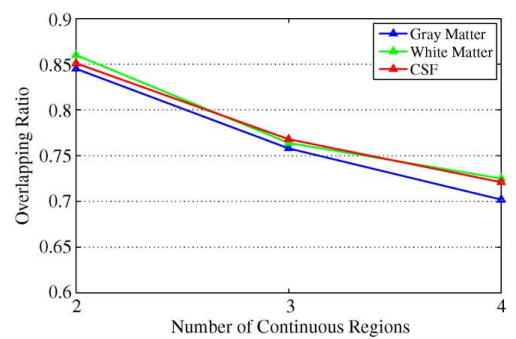

(c)

Fig. 8. Average tissue overlapping ratios of the proposed method by relaxing the number of continuous regions of the USRD feature on (a) the BrainWeb database without bias field distortion, (b) the BrainWeb database with bias field distortion, and (c) the IBSR database.

Similar to Section V-A, we also study the effect of applying the intensity standardization process before conducting registration for different registration approaches. Table VII lists the tissue overlapping ratios of different approaches after applying the intensity standardization procedure to the input images for the IBSR database.

It is shown in Table VII that the registration performances of different approaches are better after applying the intensity standardization procedure, following the same trend present in Section V-A. It is also shown that the proposed method still maintains the highest tissue overlapping ratios among all the compared methods, which demonstrate the effectiveness of the proposed approach.

The average computation time for the proposed method to register one pair of input images for the IBSR database was around $2.4 \mathrm{~h}$. The computation time of all the compared methods in the IBSR database is also listed as follows: 1) FFD took 3.1 $\mathrm{h}$; 2) Demons took $1.5 \mathrm{~h}$; 3) LHF took $4.2 \mathrm{~h}$; and 4) HAMMER took $4.6 \mathrm{~h}$.

\section{Additional Experiments on Relaxing the USRD Constraints}

Here, we study the effect of relaxing the number of continuous regions defined for the uniform pattern in a deeper way. The tissue overlapping ratio measure function used in Sections V-A and V-B is still adopted as the evaluation function, and the average tissue overlapping ratios of GM, WM, and CSF using different numbers of continuous regions of the proposed USRD feature on the BrainWeb database with and without bias field distortion and on the real 3-D IBSR database are shown in Fig. 8(a)-(c).

It is shown in Fig. 8(a)-(c) that the registration accuracy of the proposed USRD feature monotonically decrease on both the databases by relaxing the number of continuous regions defined for the uniform pattern. The main reason is that, by relaxing the constraints on the number of continuous regions for the uniform pattern definition, the patterns that are less informative and sensitive to noise are also included in the uniform pattern group for consideration. It leads to the degradation of the discriminant power and saliency of the USRD feature. Therefore, the physical meaning of the uniform pattern defined in Section II and the constraint that restricts the number of continuous regions of uniform patterns to two are justified.

\section{CONCLUSION}

In this paper, a new feature-based nonrigid image registration method has been proposed. The proposed method is based on a new type of image feature, namely, the USRD. The USRD has two kinds of complementary features, i.e., USSP and UGSP, which encode the first- and second-order voxel-wise interaction information, respectively. The USRD is rotation and monotonic gray-level transformation invariant, which are properties that are analyzed and proved in the paper. The USRD can be efficiently calculated by a few voxel-wise intensity and gradient orientation comparison operations. The USRD feature is integrated with the MRF labeling framework for driving the registration process. The discriminant power of the USRD feature is directly compared with other widely used features for image registration by using the FSC. It is shown that the proposed USRD feature has remarkably high discriminant power and is more robust against bias fields. The proposed method is evaluated on both the simulated and real 3-D image databases obtained from BrainWeb and IBSR, respectively. Experimental results demonstrate that the proposed method consistently has the highest registration accuracy among all the compared methods under different conditions.

\section{REFERENCES}

[1] K. Rohr, "Image registration based on thin plate splines and local estimates of anisotropic landmark localization uncertainties," in Proc. Conf. Med. Image Comput. Comput.-Assist. Intervention, 1998, pp. 1174-1183.

[2] P. Thompson and A. Toga, "A surface-based technique for warping three-dimensional images of the brain," IEEE Trans. Med. Imag., vol. 15, no. 4, pp. 402-417, Aug. 1996.

[3] H. Chui, L. Schultz, J. Duncan, and A. Rangarajan, "A unified feature registration method for brain mapping," in Proc. Inf. Process. Med. Imag., 2001, pp. 300-314.

[4] M. Breijl and M. Sonka, "Object localization and border detection criteria design in edge-based image segmentation: Automated learning from examples," IEEE Trans. Med. Imag., vol. 19, no. 10, pp. 973-985, Oct. 2000.

[5] R. Gan and A. Chung, "Multi-dimensional mutual information based robust image registration using maximum distance gradient magnitude," in Proc. Inf. Process. Med. Imag., 2005, pp. 210-221. 
[6] Y. Wang and L. Staib, "Boundary finding with prior shape and smoothness models," IEEE Trans. Pattern Anal. Mach. Intell., vol. 22, no. 7, pp. 738-743, Jul. 2000.

[7] P. Roland, C. Graufelds, J. Whalin, L. Ingelman, M. Andersson, A. Ledberg, J. Pedersen, S. Åkerman, A. Dabringhaus, and K. Zilles, "Human brain atlas: For high-resolution functional and anatomical mapping," Human Brain Map., vol. 1, no. 3, pp. 173-184, 1993.

[8] S. Liao and A. Chung, "Multi-modal image registration using the generalized survival exponential entropy," in Proc. Conf. Med. Image Comput. Comput.-Assist. Intervention, 2006, pp. 964-971.

[9] P. Saha, "Tensor scale: A local morphometric parameter with applications to computer vision and image processing," Comput. Vis. Image Understanding, vol. 99, no. 3, pp. 384-413, Sep. 2005.

[10] P. Saha, H. Zhang, J. Udupa, and J. Gee, "Tensor scale-based image registration," in Proc. SPIE Med. Imag., 2003, pp. 314-324.

[11] L. Nyul, J. Udupa, and P. Saha, "Incorporating a measure of local scale in voxel-based 3-d image registration," IEEE Trans. Med. Imag., vol. 22, no. 2, pp. 228-237, Feb. 2003.

[12] U. Bagci and L. Bai, "Automatic best reference slice selection for smooth volume reconstruction of a mouse brain from histological images," IEEE Trans. Med. Imag., vol. 29, no. 9, pp. 1688-1696, Sep. 2010.

[13] U. Bagci, J. Udupa, and L. Bai, "The role of intensity standardization in medical image registration," Pattern Recognit. Lett., vol. 31, no. 4, pp. 315-323, Mar. 2010.

[14] D. Shen and C. Davatzikos, "Hammer: Hierarchical attribute matching mechanism for elastic registration," IEEE Trans. Med. Imag., vol. 21, no. 11, pp. 1421-1439, Nov. 2002.

[15] G. Yang, C. Stewart, M. Sofka, and C. Tsai, "Registration of challenging image pairs: Initialization, estimation, and decision," IEEE Trans. Pattern Anal. Mach. Intell., vol. 29, no. 11, pp. 1973-1989, Nov. 2007.

[16] Z. Tu, K. Narr, P. Dollar, I. Dinov, P. M. Thompson, and A. Toga, "Brain anatomical structure segmentation by hybrid discriminative/generative models," IEEE Trans. Med. Imag., vol. 27, no. 4, pp. 495-508, Apr. 2008.

[17] L. Dirk, S. Pieter, M. Frederik, V. Dirk, and S. Paul, "Nonrigid image registration using conditional mutual information," in Proc. Inf. Process. Med. Imag., 2007, pp. 725-737.

[18] A. Souza, J. Udupa, and A. Madabushi, "Image filtering via generalized scale," Med. Image Anal., vol. 12, no. 2, pp. 87-98, Aug. 2007.

[19] S. Liao and A. Chung, "Non-rigid image registration with uniform spherical structure patterns," in Proc. Inf. Process. Med. Imag., 2009, pp. 163-175.

[20] S. Liao and A. Chung, "Non-rigid image registration with uniform gradient spherical patterns," in Proc. Conf. Med. Image Comput. Comput.Assist. Intervention, 2009, pp. 696-704.

[21] A. Fisher, The Mathematical Theory of Probabilities. New York: Macmillan, 1923.

[22] D. Rueckert, L. Sonoda, C. Hayes, D. Hill, M. Leach, and D. Hawkes, "Nonrigid registration using free-form deformations: application to breast MR images," IEEE Trans. Med. Imag., vol. 18, no. 8, pp. 712-721, Aug. 1999.

[23] J. Thirion, "Image matching as a diffusion process: An analogy with Maxwell's demons," Med. Image Anal., vol. 2, no. 3, pp. 243-260, Sep. 1998.

[24] D. Shen, "Image registration by local histogram matching," Pattern Recognit., vol. 40, no. 4, pp. 1161-1172, Apr. 2007.

[25] S. Liao and A. Chung, "Feature based non-rigid brain MR image registration with symmetric alpha stable filters," IEEE Trans. Med. Imag., vol. 29, no. 1, pp. 106-119, Jan. 2010.

[26] T. Ojala, M. Pietikainen, and T. Maenpaa, "Multiresolution gray-scale and rotation invariant texture classification with local binary patterns," IEEE Trans. Pattern Anal. Mach. Intell., vol. 24, no. 7, pp. 971-987, Jul. 2002.
[27] A. Yershova, M. LaValle, and C. Mitchell, "Generating uniform incremental grids on so(3) using the Hopf fibration," in Proc. WAFR, 2008, pp. $1-15$.

[28] W. Tang and A. Chung, "Non-rigid image registration using graphcuts," in Proc. Conf. Med. Image Comput. Comput.-Assist. Intervention, 2007, pp. 916-924.

[29] C. Thomas and J. Thomas, Elements of Information Theory. Hoboken, NJ: Wiley, 2006.

[30] S. Joshi and M. Miller, "Landmark matching via large deformation diffeomorphisms," IEEE Trans. Image Process., vol. 9, no. 8, pp. 1357-1370, Aug. 2000.

[31] M. Miller and L. Younes, "Group actions, homeomorphisms, and matching: A general framework," Int. J. Comput. Vis., vol. 41, no. 1/2, pp. 61-84, Jan./Feb. 2001.

[32] B. Yuri, V. Olga, and Z. Ramin, "Fast approximate energy minimization via graph cuts," IEEE Trans. Pattern Anal. Mach. Intell., vol. 23, no. 11, pp. 1222-1239, Nov. 2001.

[33] W. Crum, D. Rueckert, M. Jenkinson, D. Kennedy, and M. Smith, "A framework for detailed objective comparison of non-rigid registration algorithms in neuroimaging," in Proc. Conf. Med. Image Comput. Comput.-Assist. Intervention, 2004, pp. 679-686.

[34] J. Udupa, V. LeBlanc, Y. Zhuge, C. Imielinska, H. Schmidt, L. Currie, B. Hirsch, and J. Woodburn, "A framework for evaluating image segmentation algorithms," Comput. Med. Imag. Graph., vol. 30, no. 2, pp. 75-87, Mar. 2006.

[35] U. Bagci and L. Bai, "Multiresolution elastic medical image registration in standard intensity scale," in Proc. SIBGRAPI, 2007, pp. $305-312$.

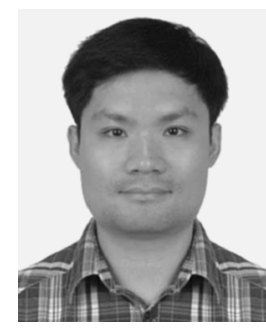

Shu Liao received the B.Eng. degree (first class honors and Academic Achievement Awards) in computer engineering, the M.Phil. degree in computer science and engineering, and the Ph.D. degree in computer science and engineering from The Hong Kong University of Science and Technology, Kowloon, Hong Kong, in 2005, 2007, and 2010, respectively.

He is currently a Postdoctoral Research Fellow in the Department of Radiology, Biomedical Research Imaging Center, University of North Carolina, Chapel Hill. His research interests include medical image analysis, texture analysis, and face recognition.

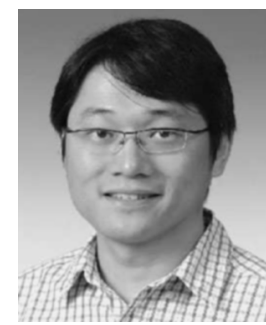

Albert C. S. Chung received the B.Eng. degree (first class honors) in computer engineering from The University of Hong Kong, Pokfulam, Hong Kong, in 1995 and the M.Phil. degree in computer science from The Hong Kong University of Science and Technology, Kowloon, Hong Kong, in 1998.

He joined the Medical Vision Laboratory, University of Oxford, Oxford, U.K., as a Doctoral Research Student with a Croucher Foundation scholarship and graduated in 2001. He was a Visiting Scientist at the Artificial Intelligence Laboratory, Massachusetts Institute of Technology, Cambridge, in 2001. He is currently an Associate Professor with the Department of Computer Science and Engineering, The Hong Kong University of Science and Technology. His research interests include medical image analysis, image processing, and computer vision.

Dr. Chung was the recipient of the 2002 British Machine Vision Association Sullivan Thesis Award for the best doctoral thesis submitted to a U.K. university in the field of computer or natural vision. 